\title{
Novel human cell expression method reveals the role and prevalence of posttranslational modification in nonmuscle tropomyosins
}

Received for publication, April 14, 2021, and in revised form, August 25, 2021 Published, Papers in Press, September 1, 2021,

https://doi.org/10.1016/j.jbc.2021.101154

Peter J. Carman ${ }^{1,2}$, Kyle R. Barrie ${ }^{1,2} \mathbb{D}$, and Roberto Dominguez

From the ${ }^{1}$ Department of Physiology, ${ }^{2}$ Biochemistry and Molecular Biophysics Graduate Group, Perelman School of Medicine, University of Pennsylvania, Philadelphia, Pennsylvania, USA

Edited by Enrique De La Cruz

Biochemical studies require large quantities of proteins, which are typically obtained using bacterial overexpression. However, the folding machinery in bacteria is inadequate for expressing many mammalian proteins, which additionally undergo posttranslational modifications (PTMs) that bacteria, yeast, or insect cells cannot perform. Many proteins also require native $\mathrm{N}$ - and $\mathrm{C}$-termini and cannot tolerate extra tag amino acids for proper function. Tropomyosin (Tpm), a coiled coil protein that decorates most actin filaments in cells, requires both native $\mathrm{N}$ - and $\mathrm{C}$-termini and PTMs, specifically $\mathrm{N}$ terminal acetylation ( $\mathrm{Nt}$-acetylation), to polymerize along actin filaments. Here, we describe a new method that combines native protein expression in human cells with an intein-based purification tag that can be precisely removed after purification. Using this method, we expressed several nonmuscle Tpm isoforms (Tpm1.6, Tpm1.7, Tpm2.1, Tpm3.1, Tpm3.2, and Tpm4.2) and the muscle isoform Tpm1.1. Proteomics analysis revealed that human-cell-expressed Tpms present various PTMs, including Nt-acetylation, Ser/Thr phosphorylation, Tyr phosphorylation, and Lys acetylation. Depending on the Tpm isoform (humans express up to $40 \mathrm{Tpm}$ isoforms), Ntacetylation occurs on either the initiator methionine or on the second residue after removal of the initiator methionine. Human-cell-expressed Tpms bind F-actin differently than their Escherichia coli-expressed counterparts, with or without Nterminal extensions intended to mimic $\mathrm{Nt}$-acetylation, and they can form heterodimers in cells and in vitro. The expression method described here reveals previously unknown features of nonmuscle Tpms and can be used in future structural and biochemical studies with Tpms and other proteins, as shown here for $\alpha$-synuclein.

Biochemical and structural studies require large amounts of pure proteins and protein complexes. When these cannot be purified from natural sources, scientists typically opt for protein expression in bacteria, and $\mathrm{N}$ - or $\mathrm{C}$-terminal affinity tags are commonly used for protein purification. These tags are often left on proteins during subsequent studies, and when they are

\footnotetext{
* For correspondence: Roberto Dominguez, droberto@pennmedicine. upenn.edu.
}

enzymatically removed, extra amino acids often remain (1). While these approaches have proven powerful and will likely continue to be used in the future, there are many circumstances in which they fail to produce accurate results. For example, most human proteins undergo multiple types of posttranslational modifications (PTMs), including acetylation, phosphorylation, and methylation (2). Such PTMs play crucial roles in the regulation of protein function, including protein stability, proteinprotein interactions, and enzymatic activity (3-6). Most PTMs present in human proteins cannot be processed by expression systems such as bacteria, yeast, or even insect cells (7-12). These expression systems may also lack the adequate machinery to fold certain mammalian proteins (13-16). Many proteins also require native $\mathrm{N}$ - and $\mathrm{C}$-termini and cannot tolerate the presence of extra amino acids from purification tags for proper function (17-21). We were confronted with these problems while studying tropomyosin $(\mathrm{Tpm})$, a protein of central importance in the actin cytoskeleton (22).

Tpm is a coiled coil dimer that polymerizes head-to-tail to form two contiguous chains, one on each side of the actin filament (F-actin). Through alternative splicing of four different genes (TPM1-4), up to 40 distinct Tpm isoforms can be expressed (23). Most isoforms are $\sim 280$-amino acids long (called long isoforms), comprising seven pseudo-repeats of $\sim 40$ amino acids, with each pseudo-repeat spanning the length of one actin subunit along the long-pitch helix of the actin filament. Long Tpms decorate the contractile actin filaments of muscle cells. In nonmuscle cells, the majority of actin filaments are also decorated with Tpm (24). Some of the genes encoding nonmuscle Tpm isoforms lack exon 2 and as a consequence such Tpm isoforms are one pseudo-repeat shorter (called short isoforms). Because the six human actin isoforms are very similar, sharing 93 to $99 \%$ sequence identity, it has been proposed that the decoration of actin filaments with distinct Tpm isoforms provides a mechanism for filaments to assume different identities and become functionally segregated in cells (25). Tpm can move azimuthally on actin, helping to regulate the interactions of most actin-binding proteins, including myosin $(26,27)$.

Although there is high sequence similarity among Tpm isoforms, gene knockout and isoform overexpression studies have 
shown that they often play nonredundant roles (22). For example, Tpm4.2 knockout causes low platelet count (28), whereas Tpm3.5 knockout causes softer, less mechanically resilient lenses in the eye (29), indicating that other Tpm isoforms cannot compensate for the lack of Tpm4.2 or Tpm3.5. Similarly, different Tpm isoforms have been implicated in processes such as muscle contraction $(30,31)$, cytokinesis (32, $33)$, embryogenesis $(34,35)$, and endocytosis (36). The temporal expression of Tpm isoforms is also tightly controlled. Thus, 16 isoforms are up- or downregulated at different stages of brain development in rodents $(37,38)$. Tpm3.1 and Tpm3.2 are found in the axon in developing neurons, whereas in mature neurons they are expressed only in cell bodies and replaced in axons by Tpm1.12 and Tpm4.2 (39).

PTMs, and in particular N-terminal acetylation (Nt-acetylation) (40-42) and phosphorylation (43-46), have been shown to critically regulate Tpm function. Proper head-to-tail assembly of Tpm coiled coils along actin filaments requires native $\mathrm{N}$ - and C-termini, i.e., Nt-acetylated and free of extra amino acids from tags $(40,47)$, whereas the phosphorylation state of Tpm can also modulate the stiffness and affinity of the head-to-tail interaction as well as the F-actin-binding affinity $(43,44,46)$. Despite the importance of these factors, previous biochemical studies have focused on one or two Tpm isoforms purified from muscle or Escherichia coli-expressed full-length and peptide fragments of Tpm isoforms that lack PTMs and native $\mathrm{N}$ - and $\mathrm{C}$-termini. While extra amino acids have been added at the $\mathrm{N}$-terminus to substitute for $\mathrm{Nt}$-acetylation $(40,41,48,49)$, it is unclear how precisely this approach mimics acetylation. Tpm coexpression with the yeast acetyltransferase NatB in $E$. coli has been also used to acetylate the $\mathrm{N}$-terminus (50). However, while yeast NatB can acetylate the $\mathrm{N}$-terminus in $E$. coli, the extent of this modification varies for different Tpm isoforms, being as low as 30\% for certain mammalian Tpm isoforms (50). Moreover, E. coli cannot add other types of PTMs (8). Furthermore, as we show here, for many Tpm isoforms the initiator methionine is removed by mammalian N-terminal aminopeptidases and Nt-acetylation occurs on the second residue. E. coli lacks the necessary regulatory and modification machineries to perform such multistep Nt-processing reactions (51). We finally show here that Nt-acetylation mimics are not always capable of recapitulating the F-actin-binding properties of native Tpm isoforms.

Here, we describe a method that combines expression in human Expi293F cells, which grow in suspension to high density and contain the native PTM machinery, with an inteinbased affinity tag that can be precisely removed through selfcleavage after purification. We applied this method to the expression of six nonmuscle Tpm isoforms and one muscle isoform (Tpm1.1). We demonstrate the general applicability of this method to other proteins by also showing expression of $\alpha$ synuclein, a protein implicated in several neurodegenerative disorders and whose function is critically regulated by numerous PTMs $(52,53)$. We performed a functional analysis of the expressed Tpms in comparison with their E. coli- expressed counterparts, revealing significant differences in their ability to bind F-actin. Further, a comprehensive proteomics analysis of the human-cell-expressed Tpms showed the type and location of various PTMs, including Nt-acetylation and phosphorylation sites. We finally show that nonmuscle Tpm isoforms can form heterodimers in vitro and in cells, as previously shown for muscle isoforms purified from tissues.

\section{Results}

\section{Human cell expression and intein-mediated purification method and its application to Tpm and a-synuclein}

The expression method developed here combines mammalian cell expression with a self-cleavable affinity tag. The cells used are Expi293F, commercialized by Thermo Fisher Scientific, which are based on human embryonic kidney (HEK) cells and have been adapted to grow in suspension to high density $(54,55)$. These cells can be transiently transfected, display high protein expression levels, and have the endogenous protein folding and PTM machineries necessary for native expression and modification of human proteins. The self-cleavable affinity tag consists of a modified intein element followed by a chitin-binding domain (CBD) and is based on the IMPACT (intein-mediated purification with an affinity chitinbinding tag) system marketed by New England Biolabs $(56,57)$. Both, $\mathrm{N}$ - and C-terminal intein-CBD tags are commercially available for bacterial protein expression, but we opted here to use a C-terminal intein-CBD tag for expression in mammalian cells to allow for $\mathrm{N}$-terminal processing and acetylation, which occurs on $\sim 90 \%$ of human proteins (58). After purification on a high-affinity chitin resin, self-cleavage of the intein takes place precisely after the last endogenous amino acid of the expressed target protein, leaving no extra amino acids from the tag. While both mammalian cell expression (59-61) and intein-CBD purification tags (62) have been previously used, to our knowledge this is the first time that the two are combined in a single method. Self-cleavage of the intein is thiolcatalyzed, meaning that a portion of the intein of bacterial origin is prematurely activated in the reducing environment of the cytoplasm of eukaryotic cells, likely explaining why they have not been used for mammalian cell expression. Yet, we show here that a significant portion of the expressed protein remains fused to the intein-CBD tag, and milligram yields of purified protein can be obtained.

We built a new vector (named pJCX4), which incorporates the Mxe GyrA intein and CBD into a mammalian-cell expression vector (Fig. $1 A$ ). The $M x e$ GyrA intein is a 198 -amino acid self-splicing protein from Mycobacterium xenopi (63). In its native form, the intein catalyzes its own excision from the precursor protein, forming a new peptide bond between the $\mathrm{N}$ and C-terminal fragments that flank the intein $(64,65)$. To harness this process for expression and purification of a target protein, the $\mathrm{N}$ - and $\mathrm{C}$-terminal fragments of the intein precursor protein are substituted respectively by the target protein and the CBD affinity tag, and two amino acids at the C-terminus 
A

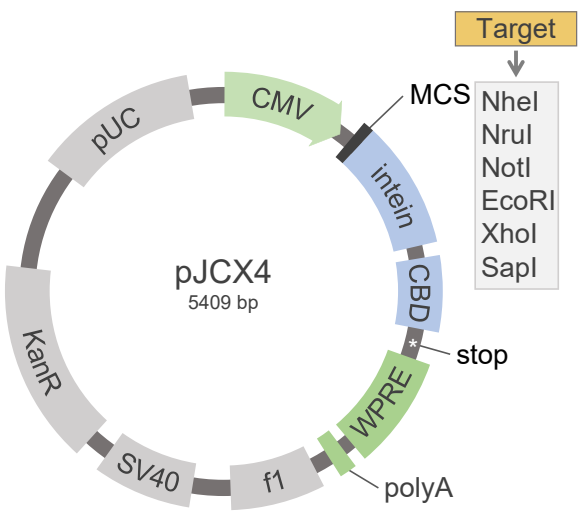

B Expression and purification steps

\begin{tabular}{|l|l|}
\hline Target Intein & CBD \\
\hline
\end{tabular}

Protein expressed in Expi293F cells is loaded onto chitin resin

\begin{tabular}{|l|l|l|l|}
\hline Target & Intein \\
Chitin \\
resin
\end{tabular}

On-column intein self-cleavage induced with reducing agent ( $\beta M E, 24 \mathrm{~h})$
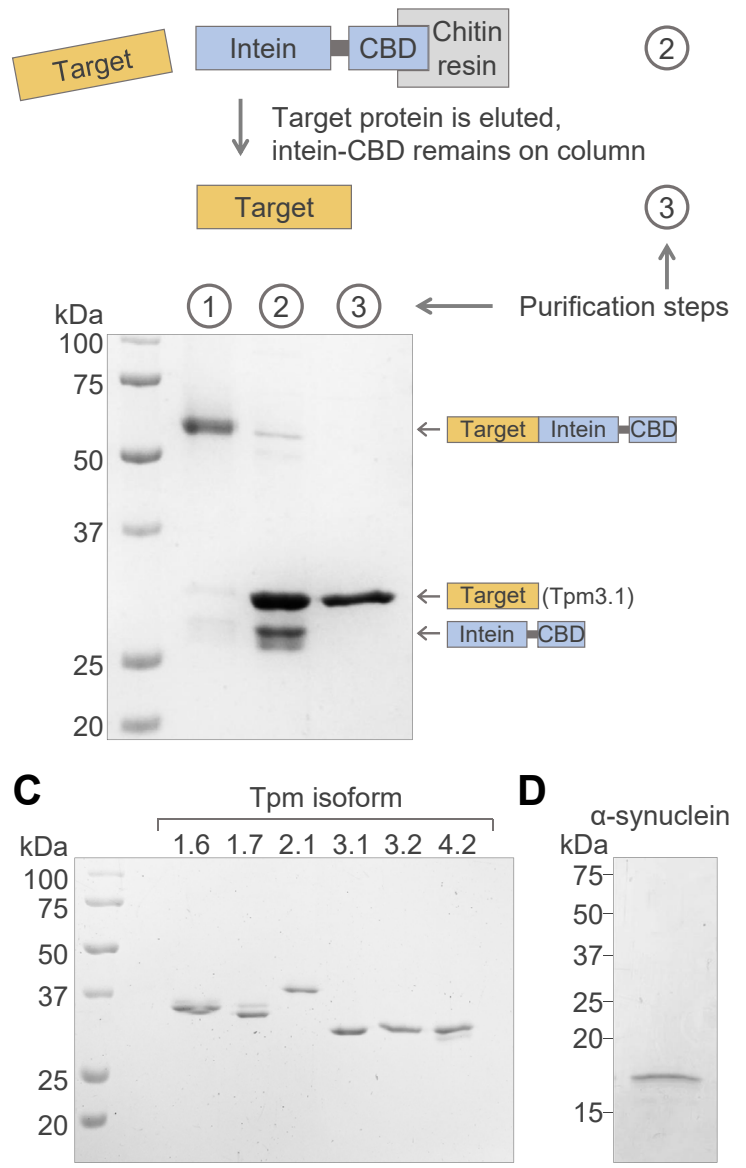

Figure 1. Cloning, expression, and purification of Tpm isoforms and asynuclein in Expi293F cells. $A$, schematic representation of the mammalian-cell expression vector designed here, featuring an intein-CBD affinity-purification and self-cleavage element. Cloning into this vector results in the expression of the target protein fused to intein-CBD (see also Fig. S1). B, schematic representation of the expression and purification steps, including purification on a chitin resin of the protein expressed in Expi293F cells, thiol-catalyzed self-cleavage of the intein using $\beta$ - of the intein moiety (Asn-Cys) are mutated to Ala-Thr, which turns the intein splicing activity into precise cleavage at the junction between the target protein and the intein (66).

In addition to the intein-CBD element, the expression vector includes the cytomegalovirus promoter (CMV) and the simian virus 40 (SV40) polyadenylation (polyA) signal, as well as the woodchuck hepatitis virus posttranscriptional regulatory element (WPRE) for enhanced expression (Fig. 1A). It also includes pUC and $\mathrm{f} 1$ origins of replication for propagation in E. coli and the SV40 origin of replication for propagation in mammalian cells. Finally, the vector contains the kanamycin resistance gene for selection in bacteria. These elements collectively allow for plasmid cloning and amplification in E. coli and expression in Expi293F cells. A number of restriction sites are available to clone the $5^{\prime}$ end of a target gene, while the SapI or SpeI sites must be used to clone the $3^{\prime}$ end, to ensure that no vector- or tag-derived amino acids remain after self-cleavage of the intein (Fig. $1 A$ and Fig. S1, $A$ and $B$ ). Growth and transfection of Expi293F cells are carried out according to the manufacturer's protocol, with minor changes (see Experimental procedures). After harvesting and lysing cells, the fusion precursor protein is loaded onto a chitin affinity resin. The high affinity of the CBD-chitin interaction allows for extensive washing, and the resin can be analyzed during washing by SDS-PAGE to monitor the removal of contaminants (Fig. $1 B$ and Fig. S1C). The intein is then activated with the addition of a reducing agent, which allows the target protein to be released from the column.

We used this method here to express and purify the six most studied nonmuscle Tpm isoforms (Fig. 1C), as well as muscle Tpm1.1 and $\alpha$-synuclein (Fig. 1D). As an example, after extensive washing on the chitin resin, the fusion protein Tpm3.1-intein-CBD is nearly contaminant free (step 1 in Fig. $1 B$ ). Self-cleavage of the intein, induced with $\beta$-mercaptoethanol for $24 \mathrm{~h}$ (step 2), is followed by elution of the target protein (step 3 ). Although the proteins were mostly pure after the intein-affinity purification step, proteins here were additionally purified by ion exchange chromatography. Final yields varied from protein to protein, ranging from 1 to $8 \mathrm{mg}$ of purified proteins per liter of Expi293F culture.

\section{Posttranslational modification of Expi293F-expressed Tpm and a-synuclein}

To evaluate the presence of PTMs and native $\mathrm{N}$ - and Ctermini in the proteins expressed here we used two approaches, tandem mass spectrometry (MS)-based proteomics and Pro-Q Diamond phosphoprotein gel staining (Thermo Fisher Scientific). While proteomics reveals both the types and sites of all forms of PTMs, fluorescent Pro-Q Diamond staining is used here as a qualitative way to assess the presence

mercaptoethanol ( $\beta \mathrm{ME})$, and elution of the target protein. As an example, SDS-PAGE analysis at the bottom of the panel illustrates the progression of these steps for Tpm3.1 (see also Fig. S1). C and D, SDS-PAGE analysis and Coomassie Brilliant Blue R-250 (BIO-RAD) staining of the six nonmuscle Tpm isoforms (panel $C$ ) and a-synuclein (panel $D$ ) expressed and purified using this strategy, and further purified using ion exchange chromatography (see Experimental procedures). 


\section{Novel mammalian cell expression and purification method}

of phosphate groups in the expressed proteins. However, this method cannot discriminate between phosphorylation on tyrosine, serine, or threonine residues and cannot reveal the location of phosphorylation sites. Because signaling cascades that get activated during cell lysis can lead to increased dephosphorylation (67), phosphatase inhibitors were used here to enhance Pro-Q Diamond staining of small proteins cultures (Fig. 2, $B$ and $D$ ). However, proteomics and functional studies were performed on the same batches of proteins, purified from larger cultures without phosphatase inhibitors.

A

Tpm3.1 MS peptide coverage map relative to the exon map


B $\mathrm{kDa}$

Tpm3.1



C

a-synuclein MS peptide coverage map relative to the protein domains

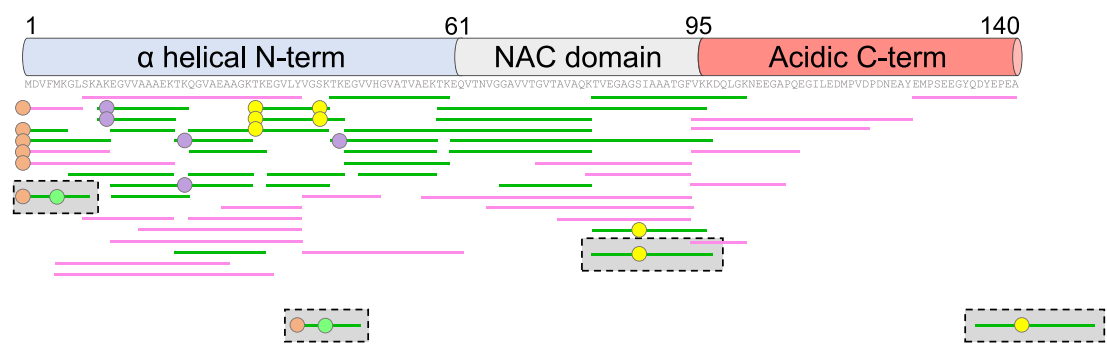

D $k \mathrm{ka}$


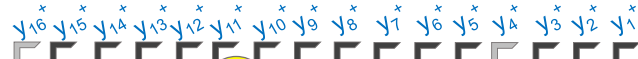
Coomassie Pro-Q

Diamond

81- TVEGA G PS I A A A T G F V K K 97

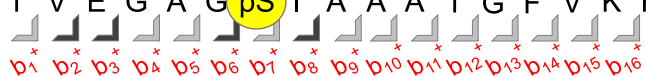
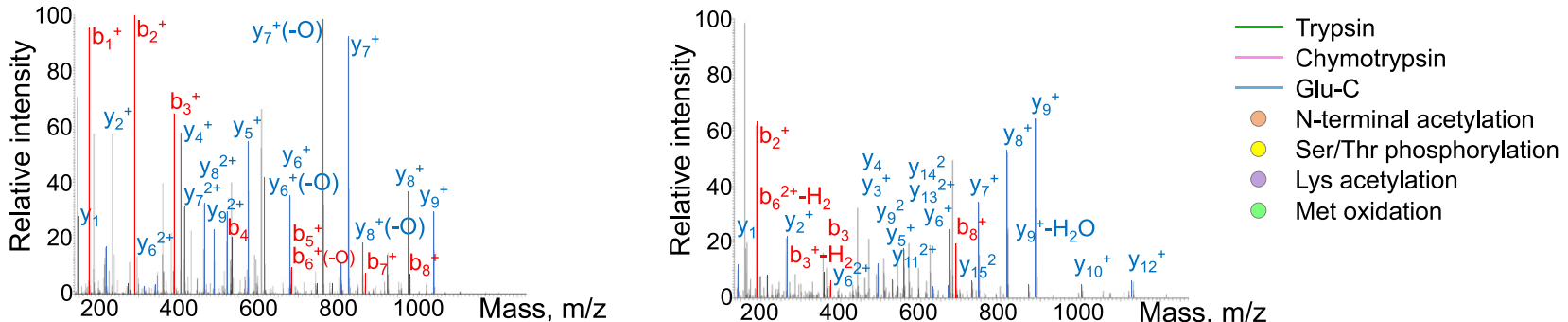

Figure 2. Posttranslational modification analysis of Expi293F-expressed Tpm3.1 and a-synuclein. $A$ and $C$, mass spectrometry peptide coverage maps of Tpm3.1 and a-synuclein shown relative to exon and protein domain diagrams, respectively. Green, magenta, and blue lines indicate peptides obtained by digestions with trypsin, chymotrypsin, and Glu-C, respectively, and whose masses were identified by mass spectrometry using a false discovery rate (FDR) threshold of $<1 \%$ (see also Table S1 and Fig. S2). Orange, yellow, red, purple, and green circles indicate Nt-acetylation, Ser/Thr phosphorylation, Tyr phosphorylation, Lys acetylation, and Met oxidation, respectively. The mass spectrometry spectra (MS2) of three representative peptides (highlighted by black dashed lines and gray background) are shown as examples, including N-terminal peptides of Tpm3.1 and a-synuclein, and a central peptide of asynuclein. These peptides show different types of PTMs (Nt-acetylation, Ser/Thr phosphorylation, and Met oxidation). The MS2 spectra show the b (Nterminal) and y (C-terminal) fragment ions detected by mass spectrometry for each peptide. Sequence diagrams corresponding to the three spectra illustrate all the theoretical fragment ions colored black (observed experimentally) or gray (nonobserved). B and D, SDS-PAGE analysis of purified samples of Tpm3.1 and a-synuclein, using phosphatase inhibitors to reduce sample dephosphorylation during purification and stained using either Coomassie Brilliant Blue R-250 (BIO-RAD) or Pro-Q Diamond Phosphoprotein Gel Stain (Thermo Fisher Scientific). 


\section{Novel mammalian cell expression and purification method}

To ensure full peptide coverage during MS analysis, the expressed proteins were subjected to digestions using three different enzymes: trypsin, chymotrypsin, and Glu-C. To establish the identity of each peptide in the observed MS spectra, their masses were matched to the peptides in a Tpm sequence database, using a false discovery rate (FDR) threshold of less than 1\% (Table S1). Full peptide coverage was obtained for all the proteins expressed, including Tpm isoforms and $\alpha-$ synuclein, with one exception, Tpm4.2, for which coverage was 99\% (Fig. 2, $A$ and $C$ and Fig. S2). This analysis revealed several interesting findings. All the proteins, including $\alpha$-synuclein, were Nt-acetylated, which as mentioned above is a precondition for optimal Tpm polymerization along actin filaments $(40,41)$. The three short Tpm isoforms analyzed (Tpm3.1, Tpm3.2, Tpm4.2), which use exon 1b (instead of exon 1a used in long isoforms), were acetylated on the second residue. In other words, for these three isoforms the initiator methionine is first removed during $\mathrm{N}$-terminal processing and $\mathrm{Nt}$-acetylation then takes place on the second residue. Different $\mathrm{N}$-terminal acetyl transferases (NATs) likely acetylate Tpm isoforms at the initiator methionine and at position 2. Based on the known sequence specificity of the various human NATs (68), we anticipate all short isoforms with $\mathrm{N}$-terminal sequence MetAla-Gly, including Tpm3.1, Tpm3.2, and Tpm4.2, have their initiator methionine removed by MetAP (methionine aminopeptidase) and then become acetylated by NatA on Ala-2. On the other hand, long isoforms with $\mathrm{N}$-terminal sequence Met-Asp-Ala, including Tpm1.6, Tpm1.7, and Tpm2.1, are acetylated by NatB on the initiator methionine. $\alpha$-Synuclein, with N-terminal sequence Met-Asp-Val is known to be acetylated on the initiator methionine by NatB (69) (Fig. 2C). For all the proteins expressed here, intact and native $\mathrm{C}$-termini peptides were also identified (Fig. 2, $A$ and $C$ and Fig. S2), confirming that self-cleavage of the intein proceeded as expected, without the removal of any endogenous amino acid of the target protein or the presence of any extra amino acid from the tag.

Numerous phosphorylation sites, including serine/threonine and tyrosine phosphorylation, were identified in our expressed proteins with a rather stringent FDR $<1 \%$ (Table 1 , Table S1, Fig. 2, Figs. S2 and S3). Other forms of PTMs were also identified, including several sites of lysine acetylation. Methionine oxidation was also observed, including, for instance, $\alpha$-synuclein Met-5 (Fig. $2 C$ and Table S1); however, this is not a native modification but rather one that occurs during sample preparation for mass spectrometry (70). Table S1 contains a complete list of all the peptides and PTMs observed and Fig. S3 shows the annotated MS2 spectra of all posttranslationally modified peptides. The PTMs identified here are mostly consistent with previous proteomics studies curated by PhosphoSitePlus (71). However, previous studies used cell lines or tissue samples containing mixtures of Tpm isoforms, which precludes us from performing isoform-specific comparisons with our data.

\section{Comparison of the activities of Expi293F- versus E. Coli- expressed Tpm isoforms}

The defining biochemical activity of Tpm is binding to Factin, which by combinatorial association of different isoforms of both Tpm and actin can give rise to a large number of Tpmactin copolymers with distinct properties and activities in cells (25). Accordingly, we used here actin cosedimentation assays to compare the actin-binding affinities of a representative group of three Expi293F-expressed Tpm non-muscle isoforms, corresponding to three different genes and comprising both short (Tpm3.1) and long (Tpm1.6 and Tpm2.1) isoforms with differently processed N-termini. Quantification of multiple such experiments, using SDS-PAGE and densitometric analysis, and fitting of the data to a Hill equation revealed the parameters of the interactions of $\mathrm{Tpm}$ isoforms with $\mathrm{F}$-actin, including the apparent dissociation constant $\left(K_{\text {app }}\right)$ and Hill coefficient (a measure of the cooperativity of the interaction) (Fig. 3, $A, B$ and $F$ and Fig. S4). This analysis confirmed binding of all three of the Tpm isoforms to F-actin, but with significantly higher affinity for Tpm1.6 $\left(K_{a p p}=0.32 \pm 0.06 \mu \mathrm{M}\right)$ than Tpm2.1 or Tpm3.1 $\left(K_{\text {app }}=1.78 \pm 0.10 \mu \mathrm{M}\right.$ and $1.72 \pm 0.09 \mu \mathrm{M}$, respectively). Interestingly, the latter two isoforms had very similar affinities for F-actin, despite one being one pseudo-repeat longer than the other and having differently processed $\mathrm{N}$-termini.

We then compared the two most studied nonmuscle isoforms (Tpm1.6 and Tpm3.1) with their E. coli-expressed counterparts, both with and without the addition at the $\mathrm{N}$-terminus of three amino acids (Met-Ala-Ser) that have been widely used to mimic acetylation $(41,72)$. This analysis revealed several interesting results. First, for the long isoform Tpm1.6, the Expi293F- and E. coli-expressed proteins had very similar affinities for F-actin when Met-Ala-Ser was added at the N-terminus (Fig. 3, $C$ and $F$ ). However, the affinity of $E$. coli-expressed Tpm1.6 dropped $\sim 15-$ fold when Met-Ala-Ser was not added. This result suggests that for Tpm1.6 the addition of Met-Ala-Ser adequately mimics Nterminal acetylation for binding to F-actin, although it remains to be shown whether this modification can fully account for other regulatory activities of Tpm1.6 in controlling the interactions of actin-binding proteins (ABPs) with the actin filament. For the

Table 1

PTMs identified in the human-cell-expressed proteins (FDR $<1 \%$ )

\begin{tabular}{llll}
\hline Protein & Nt-acetylation & \multicolumn{1}{c}{ Ser/Thr/Tyr phosphorylation } & \multicolumn{1}{c}{ Lys acetylation } \\
\hline Tpm1.6 & Met-1 & Ser-87, Ser-123, Ser-174, Ser-186, Thr-199, Ser-252 & Lys-77, Lys-118, Lys-189 \\
Tpm1.7 & Met-1 & Ser-87, Ser-174, Ser-245, Ser-252, Tyr-221 & Lys-48, Lys-152, Lys-161, Lys-226 \\
Tpm2.1 & Met-1 & Thr-79, Ser-87 & Lys-37, Lys-77 \\
Tpm3.1 & Ala-2 & Thr-5, Thr-6, Ser-51, Thr-143 & Lys-13, Lys-76, Lys-82, Lys-225 \\
Tpm3.2 & Ala-2 & Ser-51 & Lys-11, Lys-76, Lys-169 \\
Tpm4.2 & Ala-2 & Ser-6 & Lys-44, Lys-162, Lys-169, Lys-197 \\
$\alpha$-synuclein & Met-1 & Thr-33, Ser-42, Ser-87 & Lys-12, Lys-23, Lys-45 \\
\hline
\end{tabular}


A

[Ac-Tpm1.6], $\mu \mathrm{M}$, [actin] $=7 \mu \mathrm{M}$

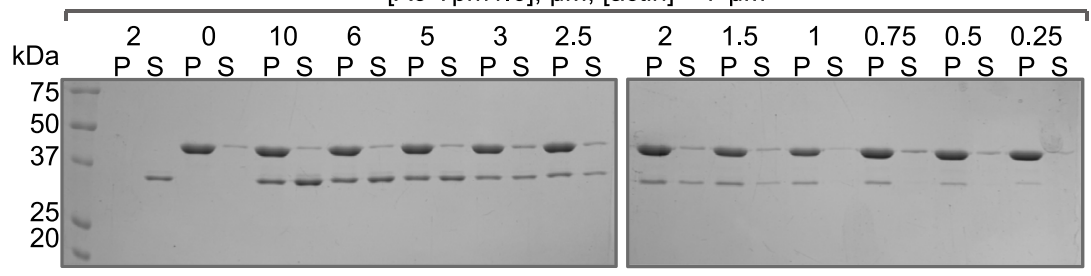

B

Expi293F-expressed Tpm isoforms

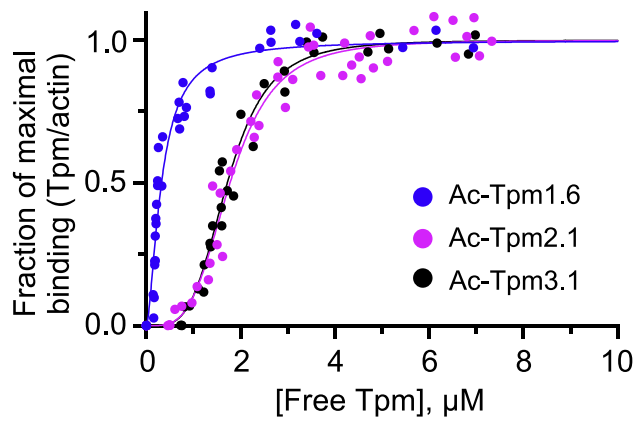

D

Tpm3.1 (Expi293F-vs. E. coli-expressed)



Tpm1.6 (Expi293F- vs. E. coli-expressed)



E

Tpm1.1 (Expi293F-vs. E. coli-expressed vs. tissue purified)

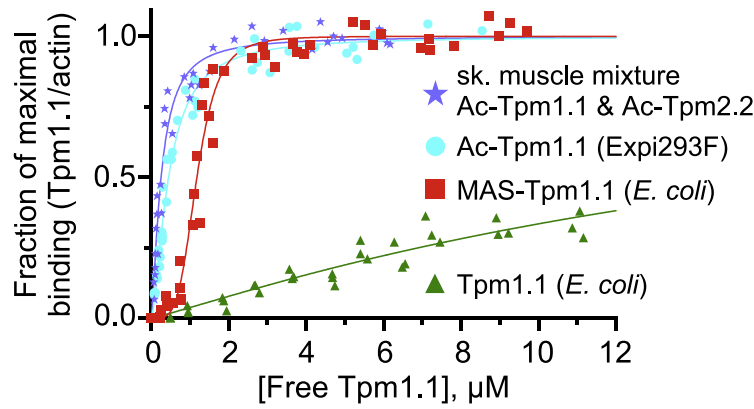

$\mathbf{F}$

\begin{tabular}{clccc}
\hline \multirow{2}{*}{ Tpm isoform } & $\begin{array}{c}\text { Expressed } \\
\text { protein }\end{array}$ & Expression cell & $K_{\text {app }}(\mu \mathrm{M})$ & Hill coefficient \\
\hline \multirow{2}{*}{ Tpm1.6 } & Ac-Tpm1.6 & Expi293F & $0.32 \pm 0.06$ & $1.64 \pm 0.45$ \\
& MAS-Tpm1.6 & E. coli & $0.31 \pm 0.07$ & $1.00 \pm 0.21$ \\
& Tpm1.6 & E. coli & $4.34 \pm 0.52$ & $2.86 \pm 0.78$ \\
\hline \multirow{2}{*}{ Tpm3.1 } & Ac-Tpm3.1 & Expi293F & $1.72 \pm 0.09$ & $3.95 \pm 0.78$ \\
& MAS-Tpm3.1 & E. coli & $2.97 \pm 0.09$ & $5.56 \pm 0.96$ \\
& Tpm3.1 & E. coli & $2.97 \pm 0.13$ & $4.34 \pm 0.59$ \\
\hline Tpm2.1 & Ac-Tpm2.1 & Expi293F & $1.78 \pm 0.10$ & $3.76 \pm 0.74$ \\
\hline Tpm1.1 & Ac-Tpm1.1 & Expi293F & $0.45 \pm 0.03$ & $1.57 \pm 0.17$ \\
& MAS-Tpm1.1 & E. coli & $1.19 \pm 0.06$ & $4.35 \pm 0.95$ \\
& Tpm1.1 & E. coli & N/A & N/A \\
\hline Muscle tissue & Ac-Tpm1.1 \& & Skeletal & $0.26 \pm 0.03$ & $1.47 \pm 0.23$ \\
& Ac-Tpm2.2 & muscle & &
\end{tabular}

Figure 3. Binding of Tpm isoforms to F-actin using cosedimentation analysis. $A$, representative SDS-PAGE cosedimentation analysis of Expi293Fexpressed Tpm1.6 $(0.25-10 \mu \mathrm{M})$ with F-actin $(7 \mu \mathrm{M})$. Controls include actin alone, found mostly in the pellet fraction (P), and Tpm1.6 alone, found exclusively in the supernatant fraction (S). $B$, between three and four cosedimentation experiments were performed for each of the three Tpm isoforms analyzed (Tpm1.6, Tpm2.1, Tpm3.1; color-coded), the density of the bands in the gels was quantified and data were plotted as the fraction of maximal binding versus the free concentration of each Tpm isoform (see Fig. S4 and Experimental procedures). The data were fit to a Hill equation to obtain the dissociation constant $\left(K_{a p p}\right)$ and Hill coefficient of each interaction. $C-E$, experiments similar to those shown in panels $A$ and $B$ were performed to compare the F-actin-binding affinities of Expi293F-expressed Tpm1.6, Tpm3.1, and Tpm1.1 with their E. coli-expressed counterparts, with and without the N-terminal addition of Met-Ala-Ser (MAS), a modification thought to mimic acetylation $(41,72)$. Panel $(E)$ also includes analysis of the Tpm mixture purified from rabbit skeletal muscle. $F$, parameters of the fits shown in panels $B-E$, including $K_{a p p}$ and Hill coefficient. 


\section{Novel mammalian cell expression and purification method}

short isoform Tpm3.1 the two $E$. coli-expressed proteins, with and without Met-Ala-Ser at the N-terminus, had nearly identical affinities for F-actin, but lower than the affinity of Expi293Fexpressed Tpm3.1 (Fig. 3, D and F). Therefore, for Tpm3.1 the addition of extra amino acids at the $\mathrm{N}$-terminus cannot substitute for native $\mathrm{Nt}$-acetylation. We suspect this behavior may affect other Tpm isoforms, which is an important consideration since many studies have used extra N-terminal amino acids to substitute for Nt-acetylation irrespective of isoform.

We asked whether the ability of extra amino acids to mimic Nt-acetylation depended on the specific exon usage of each isoform. Tpm1.6 and Tpm3.1 use exons $1 \mathrm{a}$ and $1 \mathrm{~b}$, respectively, which leads to substantial differences in sequence at their N-termini that could affect the head-to-tail interaction (Fig. S5). To test this possibility, we analyzed the most abundant muscle isoform, Tpm1.1, which has been extensively investigated and like Tpm1.6 uses exon 1a. As previously observed (49), E. coli-expressed Tpm1.1 cosedimented very poorly with F-actin (Fig. 3, $E$ and $F$ ). While the addition of Met-Ala-Ser at the N-terminus restored most of its F-actinbinding affinity, the affinity was even higher ( $\sim 2.6$-fold) for Expi293F-expressed Tpm1.1. In this case, we can compare these numbers to muscle tissue-purified Tpm, consisting of a mixture of isoforms Tpm1.1 and Tpm2.2 (73). We found that the F-actin-binding affinity of Expi293F-expressed Tpm1.1 is strikingly similar to that of the tissue-purified Tpm1.1/Tpm2.2 mixture (Fig. 3, $E$ and $F$ ).

It is known that most Tpm isoforms bind cooperatively to Factin (74-76). Here, the isoforms that bound F-actin with lower affinity displayed higher cooperativity, and this was true among different isoforms as well as among variants of the same isoform expressed in different cell types. Thus, Tpm2.1 and Tpm3.1 exhibited higher cooperativity than Tpm1.6 and Tpm1.1, whereas the E. coli-expressed Tpm isoforms that bound F-actin with lower affinity also showed higher cooperativity (Fig. 3F). We note, however, that the cooperativity of Tpm isoforms that bind F-actin with higher affinity may be somewhat underestimated, due to the inability to perform gel densitometry at samples concentrations $<0.25 \mu \mathrm{M}$ (the lowest Tpm concentration used here).

\section{Nonmuscle Tpm isoforms can form heterodimers}

The actin filaments of the contractile apparatus of muscle cells are decorated with two or more Tpm isoforms, which often associate as heterodimers $(73,77-79)$. For instance, in skeletal muscle Tpm1.1/Tpm2.2 (also known as isoforms $\alpha$ and $\beta$ ) heterodimers form more favorably than Tpm1.1/ Tpm1.1 homodimers (73). It is unknown whether nonmuscle Tpm isoforms can also form heterodimers in cells.

To explore this question, we modified the mammalian cell expression system described here to coexpress pairs of Tpm isoforms. We designed a new mammalian cell expression vector, containing an internal ribosomal entry site (IRES) inserted in between two multicloning sites (MCS), one for each Tpm isoform being coexpressed (Fig. 4A). The use of the coexpression IRES vector, instead of cotransfection with two separate vectors, ensures that similar amounts of the two Tpm isoforms are expressed together in time and space. Adjacent to MCS1 and MCS2, the vector contains a V5 and a FLAG affinity tag, respectively. In this way, the coexpressed Tpm isoforms have native N-termini, but either V5 or FLAG affinity tags added at the $\mathrm{C}$-terminus. These tags are used for tandem affinity purification and detection via Western blot analysis (Fig. 4B), a method we previously used to study wild-type/ mutant heterodimers of IRSp53 (80). The presence of tags likely disrupts the head-to-tail interaction of Tpm along Factin, but because coiled coil formation does not depend on Tpm's ability to bind F-actin, these tags were not removed for the experiments described here. When two Tpm isoforms are coexpressed using this strategy, they can form two independent homodimers, heterodimers, or a combination of homodimers and heterodimers. To discriminate among these possibilities, we purified the mixture on a FLAG resin, washed extensively, and the eluted sample was then analyzed by antiFLAG and anti-V5 Western blotting (Fig. 4B). The presence of an anti-V5 signal in the eluted sample (but not in the last wash) is a strong indication of heterodimer formation. This procedure is illustrated here for the coexpressed isoform pair Tpm3.1-V5/Tpm4.2-FLAG (Fig. 4C). Note that the anti-FLAG and anti-V5 signals disappear almost entirely in the last wash, but both signals show up strongly in the eluted sample, indicating that any Tpm3.1-V5 that was retained in the FLAG resin was forming heterodimers with Tpm4.2-FLAG.

Because heterodimerization of long muscle isoforms has been demonstrated $(73,77,81)$, the analysis here focused on four short, nonmuscle Tpm isoforms: Tpm3.1, Tpm3.2, Tpm3.5, and Tpm4.2. Note that Tpm isoforms of different lengths are unlikely to heterodimerize since this would lead to parts of the hydrophobic core of the coiled coil being exposed to the solvent. We coexpressed the following pairs: Tpm3.1V5/Tpm3.2-FLAG, Tpm3.1-V5/Tpm4.2-FLAG, and Tpm3.5V5/Tpm4.2-FLAG, which all formed heterodimers (Fig. 4D). To control for potential differences in the expression levels of the proteins cloned into MCS1 and MCS2, we switched the order of isoforms and coexpressed the pair Tpm3.2-V5/ Tpm3.1-FLAG, which also formed a heterodimer. Another control showed that coexpressed Tpm3.1-V5/Tpm3.1-FLAG as well as Tpm3.2-V5/Tpm3.2-FLAG form homodimers despite the different tags. Finally, Tpm3.1-FLAG cloned alone into MCS2 (MCS1 empty) also eluted as a homodimer and no anti-V5 signal was detected in the eluted sample (Fig. 4D).

These results strongly suggest that Tpm3.1, Tpm3.2, Tpm3.5, and Tpm4.2, and likely other nonmuscle isoforms can form heterodimers as well as homodimers in cells when coexpressed together. We then asked whether they would preferentially reassociate as homodimers or heterodimers after boiling in vitro, which unfolds the coiled coil, as shown for muscle isoforms (82-84). For this, we modified the procedure described above introducing two additional steps; after purification on the FLAG resin, the eluted samples were boiled, then allowed to slowly refold during cooling, repurified on a V5 resin, and then analyzed by anti-FLAG and anti-V5 Western blotting (Fig. $4 E$ ). In this manner, we analyzed the 
Novel mammalian cell expression and purification method

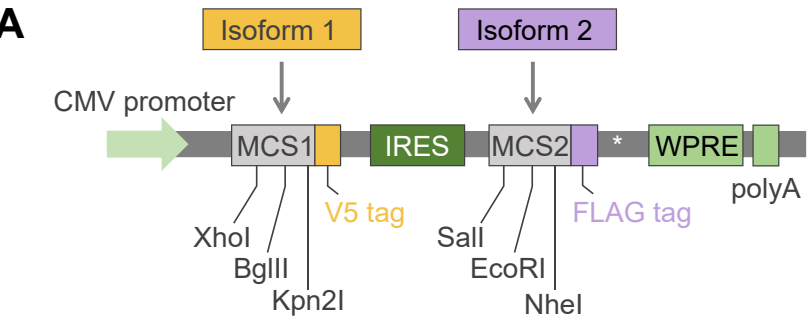
B Two coexpressed Possible outcomes Analysis strategy
Tpm isoforms
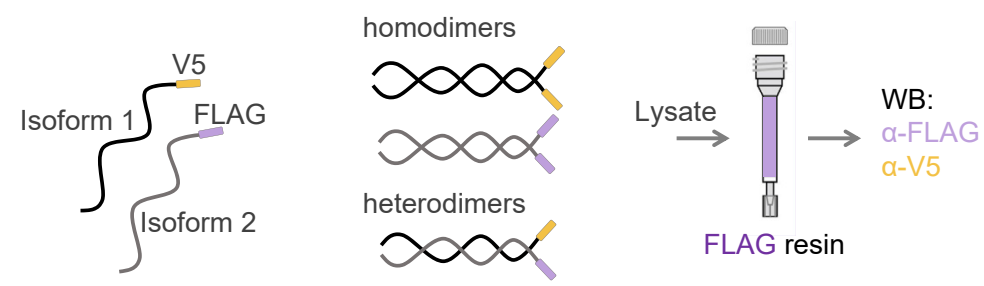

FLAG resin

C
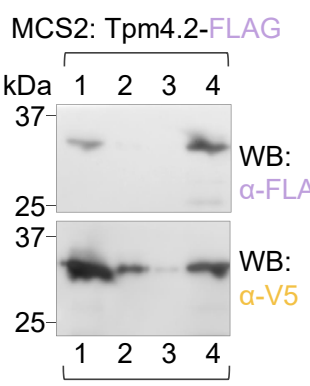

MCS1: Tpm3.1-V5
D

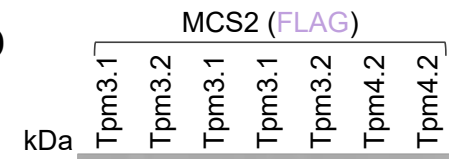

$\mathrm{kDa}$

1. Lysate

2. $1^{\text {st }}$ wash

3. Last wash

4. FLAG elution

30

-

$40-$

30



WB:

$\alpha-F L A G$

WB:

$\mathbf{E}$



$\mathbf{F}$

1. Lysate

2. Flowthrough

Step 1

3. FLAG $1^{\text {st }}$ wash

4. FLAG last wash

5. FLAG elution

Step 2 boil/cool

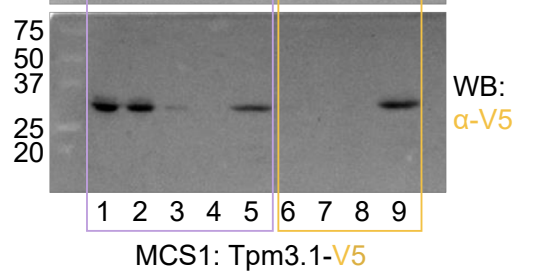

Step 3

6. V5 flowthrough

7. V5 $1^{\text {st }}$ wash

8. V5 last wash

9. V5 elution

Figure 4. Heterodimerization of nonmuscle Tpm isoforms. $A$, schematic representation of the MCS1-IRES-MCS2 elements of vector pJC8 used for coexpression of pairs of Tpm isoforms, including a depiction of the restriction sites and affinity tags (FLAG and V5) associated with each MCS. B, illustration of the potential association of coexpressed Tpm pairs as homodimers or heterodimers and strategy for the FLAG tag-based purification and Western blotbased detection of such species using anti-FLAG and anti-V5 antibodies. C, illustration of this strategy for the coexpressed pair of Tpm3.1-V5 and Tpm4.2FLAG, showing the presence of both isoforms, i.e., heterodimers reactive to both anti-FLAG and anti-V 5 antibodies, in the elution sample after extensive washing on the FLAG resin. $D$, Western blot analysis of the FLAG elution samples from seven such experiments, including six Tpm pairs and a control experiment with Tpm3.1-FLAG cloned into MCS2 and no Tpm isoform cloned into MCS1. E, experimental design for the detection of Tpm heterodimers after unfolding and refolding. Following the FLAG elution, samples are boiled and cooled and then purified on a V5 resin. $F$, illustration of this strategy for the coexpressed pair of Tpm3.1-V5 and Tpm3.2-FLAG. After extensive washing, Western blot detection of anti-FLAG and anti-V5 signals in the sample from the V5 resin demonstrates that Tpm3.1/Tpm3.2 heterodimers formed spontaneously during refolding. 


\section{Novel mammalian cell expression and purification method}

pair Tpm3.1-V5/Tpm3.2-FLAG and monitored each step of this procedure by Western blotting (Fig. 4F). The initial steps of the procedure (i.e., purification on the FLAG resin) confirmed the formation of heterodimers, as described above (Fig. 4D). After boiling/cooling the sample was loaded onto the V5 resin. The flowthrough from the V5 resin reacted only with the anti-FLAG antibody, indicating that some of the heterodimers had reassociated as homodimers. Yet, the V5 elution showed strong anti-FLAG and anti-V5 signals. Any Tpm3.2FLAG retained in the V5 resin was likely forming heterodimers with Tpm3.1-V5. Together, the data presented here show that nonmuscle Tpm isoforms can form both homodimers and heterodimers in cells and in vitro.

\section{Discussion}

\section{Main features and general applicability of the expression-} purification method

We have developed a novel method for protein expression and purification that integrates the advantages of mammalian cell expression with those of an intein-CBD purification tag. The mammalian cells used, Expi293F, grow to high density in suspension and are adapted for high-yield protein expression. More importantly, these cells naturally contain the necessary folding and PTM machineries to produce fully native human proteins. The intein-CBD tag, on the other hand, provides three key advantages. First, it is a high-affinity tag that allows for extensive on-column washing, such that high protein purity can be achieved in a single purification step. Second, this is a self-cleavable tag, which can be activated on-column, bypassing the necessity for enzymatic cleavage after purification and additional purification associated with other tags that can in some cases produce nonspecific cleavage and introduces two new contaminants, i.e., the cleavage enzyme and the released affinity tag. Third, through proper design of the cloning primers (as shown in Fig. S1), self-cleavage of the intein can take place precisely after the last C-terminal amino acid of the expressed protein, without the deletion of endogenous amino acids or the presence of extra amino acids from the tag. One potential drawback of this system is that a certain amount of premature cleavage may take place in the reducing environment of the cytoplasm of Expi293F cells, which ultimately reduces protein yields. Yet, for all the proteins expressed here yields of $\sim 1 \mathrm{mg}$ of highly pure proteins were obtained from relatively small Expi293F cultures of $250 \mathrm{ml}$. Such protein amounts are clearly sufficient for most biochemical and structural studies and the technology is cost permissive for most laboratories possessing a mammalian cell incubator. The combined benefits and relative simplicity of this method make it easily adaptable for the expression of most human proteins and particularly those whose activities depend upon key PTMs. Thus, while the initial motivation for this work was the expression of several cytoskeletal proteins of interest to our laboratory, such as Tpm, we extended it here to the expression of $\alpha$-synuclein, a protein of intensive interest and a notable target of many PTMs $(52,53)$. To facilitate the adoption of this system, we deposited the two vectors developed here with the Addgene plasmid repository (www. addgene.org, plasmid IDs 170756 and 170757).

\section{PTMs of human cell-expressed nonmuscle Tpm isoforms}

The use of the human-cell-expression system described here allowed us to make several discoveries. One discovery concerns the presence of numerous forms of PTMs, including Ntacetylation, Ser/Thr and Tyr phosphorylation, and Lys acetylation in nonmuscle Tpm isoforms (Fig. 2A, Figs. S2 and S3, Table 1). We have limited the list of PTMs reported here to sites detected with very high fidelity (FDR $<1 \%$ ), but many other sites were detected with relatively high confidence (FDR $<5 \%$ ) and could possibly play functional roles (Table S1). The importance of Ser/Thr phosphorylation in muscle Tpm isoforms has long been recognized $(85,86)$, and a more recent study suggests that phosphorylation of Ser-283 near the C-terminus of striated muscle Tpm (Tpm1.1) enhances the stiffness of the head-to-tail interaction (43). Little, however, is known about the role of Ser/Thr phosphorylation in nonmuscle Tpm isoforms. Curiously, among the phosphorylation sites observed here, several occur near the $\mathrm{N}$ - and/ or C-terminal ends and could possibly have a similar role as in muscle Tpm1.1, by modulating the stiffness of the head-to-tail interaction. To our knowledge, the role of Tyr phosphorylation in Tpm isoforms remains unexplored, and our finding here of a Tyr phosphorylation site in Tpm1.7 opens an interesting new line of inquire to understand the role of this modification. Lys acetylation of Tpm isoforms is also underappreciated. One of the Lys acetylation sites detected here occurs near the $\mathrm{N}$-terminus of Tpm3.1 and could also modulate the polymerizability of this isoform along actin filaments, as in vitro studies have suggested for muscle Tpm (87). A recent study also finds that Lys acetylation of F-actin decreases Tpm-dependent inhibition of actomyosin activity (88). Lys acetylation of nonmuscle Tpm isoforms could have a similar effect, by modulating the interactions of ABPs. The presence and extent of Nt-acetylation among nonmuscle Tpm isoforms are to this day questioned (89). Since $\sim 90 \%$ of human proteins are Nt-acetylated (58) and Nt-acetylation plays a crucial role in the polymerization of muscle Tpm isoforms (41), it is not surprising that we found here that all the nonmuscle isoforms analyzed as well as Tpm1.1 were Nt-acetylated. Moreover, not a single unacetylated $\mathrm{N}$-terminal peptide was detected, suggesting that $\mathrm{Nt}$ acetylation is constitutive (i.e., not partial). More surprising, but consistent with the known sequence specificity of human NATs (68), was the observation that the short Tpm isoforms (Tpm3.1, Tpm3.2, Tpm4.2) undergo Nt-acetylation on the second residue, after removal of the initiator Met by MetAP, which the PTM machinery of Expi293F cells was also able to achieve for these overexpressed proteins. These three isoforms are likely Nt-acetylated by NatA, whereas the long isoforms (Tpm1.6, Tpm1.7, Tpm2.1) are likely Nt-acetylated by NatB on the initiator Met (68). Some of the PTMs detected here could impact Tpm's interactions with F-actin or actin-binding proteins, which could be addressed in future structural studies. 


\section{Novel mammalian cell expression and purification method}

\section{Different activities of Tpm isoforms expressed in human and E. coli cells}

The vast majority of in vitro studies on Tpm isoforms have used E. coli-expressed proteins. For years, these studies have used the addition of extra amino acids at the N-terminus of Tpm as a way to mimic Nt-acetylation $(40,41,48,49)$ and have assumed that this modification fully replicates the natural capacity of Tpm to polymerize along actin filaments. By comparing for the first time side by side the F-actin-binding activity of naturally acetylated and E. coli-expressed Tpm isoforms with or without Nt-acetylation-mimics, we have shown here that this assumption is not always true. Thus, while the N-terminal addition of Met-Ala-Ser restores the Factin-binding affinity of E. coli-expressed Tpm1.6, it fails to do the same for Tpm3.1 and Tpm1.1 (Fig. 3). Tpm3.1 is a short isoform, whereas Tpm1.6 is a long isoform and interacts with one more actin protomer along F-actin. While this difference could possibly explain their different behaviors in these experiments, it appears prudent to test this effect individually for each Tpm isoform, particularly that this work provides a solution to the problem of $\mathrm{Nt}$-acetylation. Indeed, Tpm2.1 is also a long isoform, but we found its affinity for F-actin to be very similar to that of Tpm3.1 (Fig. 3B). We thus conclude that the affinity of a particular Tpm isoform for F-actin may depend on multiple factors, including its primary sequence and specific interactions with F-actin, the number of pseudo-repeats, and the strength of the head-to-tail interaction achieved via Ntacetylation. We also noticed an interesting correlation between affinity and cooperativity; lower Tpm binding affinity for F-actin correlates with higher cooperativity of the interaction, both among naturally occurring isoforms or within a single isoform with different head-to-tail interaction sequences (Fig. 3F). Although we could not find a previous reference to this correlation, it seems plausible that as the affinity of Tpm dimers for $\mathrm{F}$-actin becomes lower, binding becomes increasingly dependent on Tpm head-to-tail prepolymerization. This is also why a strong head-to-tail interaction, which Ntacetylation favors, enhances binding to F-actin.

\section{Nonmuscle Tpm isoforms can form heterodimers in vitro and in cells}

It has long been known that muscle Tpm isoforms function as a mixture of homodimers and heterodimers (73). Similarly, we have shown here that nonmuscle Tpm isoforms purify from cells as both homodimers and heterodimers and that their ability to heterodimerize is retained after boiling and cooling in vitro, with no apparent preference for one form of association versus another. This is a consequential finding, since Tpm heterodimerization can further expand the range and functional diversity of Tpm-actin copolymers with segregated localization and function in cells. The prevalence of heterodimers in cells could be dictated by the time, location, and amount of expression of one isoform versus another. Tpm heterodimerization is consistent with cellular studies colocalizing Tpm isoforms at specific F-actin structures. For example, Tpm3.1, Tpm3.2, and Tpm4.2 colocalized to ventral stress fibers in U2OS cells, whereas Tpm3.1 and Tpm3.2 colocalize at focal adhesions anchoring dorsal stress fibers (72). Another study found Tpm3.1 and Tpm4.2 on the actin rings of nascent endosomes in neurosecretory cells (90). Isoforms Tpm3.1, Tpm3.2, and Tpm4.2 were found here to heterodimerize, raising the possibility that their colocalization is due to heterodimerization.

In summary, we have developed a novel method for the expression of fully native proteins in human cells. The applicability of this method has been demonstrated here with two types of proteins of general interest, Tpm and $\alpha$-synuclein, whose functions are intimately linked to PTMs. Using this method, we were able to study the F-actin-binding properties of nonmuscle Tpm isoforms, discovered their ability to heterodimerize, and established the type and location of multiple PTMs. Finally, our newly developed vectors have been made available to the scientific community to facilitate the adoption of the expression method described here.

\section{Experimental procedures}

\section{Human cell expression vectors}

Vector pJCX4

The QuickChange site-directed mutagenesis kit (Agilent Technologies) was used to remove two SapI restriction sites from vector pEGFP-C1 (Takara Bio USA). A Woodchuck hepatitis virus (WHP) posttranscriptional regulatory element (WPRE) was added between the XbaI and MfeI sites of the multicloning site (MCS). The MCS-intein-chitin-binding domain of vector pTXB1 (New England Biolabs) was then inserted between sites NheI and Kpn2I, and the MCS of vector pEGFP-C1 was removed by annealed oligos, which were ligated between sites Kpn2I and XbaI. The vector is depicted in Figure $1 A$.

\section{Vector pJC8}

The intein-CBD cassette was removed from vector pJCX4 and replaced with an internal ribosome entry site (IRES) element between sites BamHI and BspE1. Two new multicloning sites, one containing a V5- and the other a FLAGaffinity tag, were introduced on either side of the IRES using annealing oligos. The V5 and FLAG epitope tags provide clear signals by Western blot analysis and are used here to recognize the expressed Tpm isoforms. The MCSs and IRES region of the vector are depicted in Figure 4A.

\section{Cloning of Tpm isoforms and a-synuclein}

The following cDNAs were used in cloning (NCBI codes): Tpm1.6 (NM_001018004), Tpm1.7 (NM_001018006), Tpm2.1 (NM_213674), Tpm3.1 (NM_153649), Tpm3.2 (NM_0010 43351), Tpm4.2 (NM_003290), $\alpha$-synuclein (NM_000345.4). The cDNAs were cloned into vector PJCX4 by PCR-amplification with primers that included the NotI $\left(5^{\prime}\right)$ and SapI $\left(3^{\prime}\right)$ restriction enzyme sites (Fig. 1 and Fig. S1). The genes encoding isoforms Tpm1.6 and Tpm3.1 with added N-terminal Met-AlaSer in vector pBAT4 were a gift from Dr Pekka Lappalainen. 
The two isoforms were also cloned without N-terminal extensions into vector $\mathrm{PBAT} 4$ by PCR-amplification with primers that included the $\mathrm{NcoI}\left(5^{\prime}\right)$ and either BamHI or XhoI $\left(3^{\prime}\right)$ restriction enzyme sites. Four of the Tpm isoforms (Tpm3.1, Tpm3.2, Tpm3.5, Tpm4.2) were separately cloned as pairs into the IREScontaining vector pJC8 (see above) using PCR primers, which included XhoI and BspEI (MCS1) or NotI and NheI (MCS2) on their overhangs. For this, the cDNA encoding isoform Tpm3.5 was generated starting from Tpm3.1 (amino acids 1-222), and the nucleotides encoding for the $\mathrm{C}$-terminal 26 amino acids that differ among these two isoforms were added by overlap extension PCR. All the primers used in this study are listed in Table S2.

\section{Protein expression in Expi293F cells and intein-based purification}

Expression was carried out with minor changes to the manufacture's protocol for the Expi293 Expression System Kit (Thermo Fisher Scientific). Expi293F cells were grown in Expi293 media to a density of $2.5 \times 10^{6}$ cells $/ \mathrm{ml}$. Cultures were incubated on an orbital shaker $(125 \mathrm{rpm})$ at $37{ }^{\circ} \mathrm{C}$ using an Isotemp incubator (Thermo Fisher Scientific) with a humidified atmosphere of $8 \% \mathrm{CO}_{2}$. Pure plasmid DNA ( $1 \mu \mathrm{g}$ per $\mathrm{ml}$ of Expi293F culture) and polyethylenimine (PEI, $3 \mu \mathrm{l}$ per $\mathrm{ml}$ of Expi293F culture) were added to separate $5 \mathrm{ml}$ aliquots of 37 ${ }^{\circ} \mathrm{C}$ Opti-MEM (Thermo Fisher Scientific), mixed after 5 min, and incubated for $30 \mathrm{~min}$ at $25{ }^{\circ} \mathrm{C}$ and then added to the Expi293F cell culture. Cultures were grown for 48 to $72 \mathrm{~h}$, optimized for each protein. Cells were harvested by centrifugation $(4000 \mathrm{~g})$ at $4{ }^{\circ} \mathrm{C}$ for $10 \mathrm{~min}$.

For purification, cell pellets were resuspended in 20 to $35 \mathrm{ml}$ of lysis buffer (20 mM HEPES pH 7.5, $500 \mathrm{mM} \mathrm{NaCl}, 1 \mathrm{mM}$ DTT). Cells were lysed using a Dounce glass piston homogenizer (Kontes Glass) and the supernatants were clarified by centrifugation at $48,000 \mathrm{~g}$ at $4{ }^{\circ} \mathrm{C}$ for $20 \mathrm{~min}$. The clarified supernatants were loaded onto $15 \mathrm{ml}$ chitin resin (packed into a column) pre-equilibrated with lysis buffer and washed extensively. Self-cleavage of the intein was activated with the addition of $50 \mathrm{mM} \beta$-mercaptoethanol at $25{ }^{\circ} \mathrm{C}$ for $24 \mathrm{~h}$. The expressed proteins were then eluted with lysis buffer, dialyzed overnight into A-buffer (20 mM HEPES pH 7.5, $50 \mathrm{mM} \mathrm{NaCl}$ ), and further purified on a Source $\mathrm{Q}$ column (MilliporeSigma) using a 50 to $300 \mathrm{mM} \mathrm{NaCl}$ gradient. Peak fractions were dialyzed into storage buffer (20 mM HEPES pH 7.5, $50 \mathrm{mM}$ $\mathrm{NaCl}$ ), flash frozen in liquid nitrogen, and stored at $-80^{\circ} \mathrm{C}$.

Phosphatase inhibitors were used to enhance the signal of phosphorylated proteins in Pro-Q Diamond phosphoprotein gel staining analysis. Calyculin A (50 nM, LC Laboratories) was added to cell cultures $30 \mathrm{~min}$ prior to harvesting. During cell lysis and purification steps, buffers were supplemented with $10 \mathrm{mM} \mathrm{NaF}, 1 \mathrm{mM} \mathrm{Na} \mathrm{VO}_{4}, 10 \mathrm{mM}$ sodium pyrophosphate, and $10 \mathrm{mM} \beta$-glycerophosphate.

\section{E. coli expression and purification of Tpm isoforms}

Tpm isoforms Tpm1.6 and Tpm3.1 (with and without MetAla-Ser at the N-terminus) were expressed in ArcticExpress(DE3) RIL cells (Agilent Technologies), grown in Terrific
Broth (TB) medium for $6 \mathrm{~h}$ at $37^{\circ} \mathrm{C}$ to an optical density of $\sim 1.5$ to 2 at $600 \mathrm{~nm}\left(\mathrm{OD}_{600}\right)$, followed by $24 \mathrm{~h}$ at $10^{\circ} \mathrm{C}$ in with $0.4 \mathrm{mM}$ isopropyl- $\beta$-D-thiogalactoside (IPTG). Cells were harvested by centrifugation $(4000 g)$ and resuspended in $20 \mathrm{mM}$ HEPES ( $\mathrm{pH} 7.5$ ), $100 \mathrm{mM} \mathrm{NaCl}, 1 \mathrm{mM}$ EDTA, and $1 \mathrm{mM}$ phenylmethanesulfonyl fluoride (PMSF). Cells were boiled in a water bath for $10 \mathrm{~min}$, cooled on ice for $10 \mathrm{~min}$, and clarified by centrifugation at $48,000 \mathrm{~g}$ for $20 \mathrm{~min}$ at $4{ }^{\circ} \mathrm{C}$. Sodium acetate ( $1 \mathrm{M}, \mathrm{pH} 4.5)$ was added to the clarified supernatant containing the Tpm isoforms (that are heat-stable) until the solution reached the isoelectric point for precipitation of each isoform (4.5-4.7). The solutions were then centrifuged at $48,000 \mathrm{~g}$ to pellet the Tpm isoforms. Pellets were dissolved in A-buffer (20 mM HEPES pH 7.5, $100 \mathrm{mM} \mathrm{NaCl}$ ) and dialyzed in the same buffer overnight. Proteins were loaded on Source $\mathrm{Q}$ ion exchange (MilliporeSigma) and eluted with a 100 to $500 \mathrm{mM} \mathrm{NaCl}$ gradient. Peak fractions containing pure proteins were dialyzed in storage buffer (20 mM HEPES pH 7.5, $50 \mathrm{mM} \mathrm{NaCl}$ ), flash frozen in liquid nitrogen, and stored at $-80{ }^{\circ} \mathrm{C}$.

\section{Preparation of samples for proteomics analysis}

Gel bands of the purified proteins were cut and destained with $100 \mathrm{mM}$ ammonium bicarbonate/acetonitrile (50:50). The bands were reduced in $10 \mathrm{mM}$ dithiothreitol and $100 \mathrm{mM}$ ammonium bicarbonate for $60 \mathrm{~min}$ at $52{ }^{\circ} \mathrm{C}$. The bands were then alkylated with $50 \mathrm{mM}$ iodoacetamide and $100 \mathrm{mM}$ ammonium bicarbonate at $25{ }^{\circ} \mathrm{C}$ for $1 \mathrm{~h}$ in the dark. The proteins were digested with enzymes (trypsin, chymotrypsin, or Glu-C) at $37^{\circ} \mathrm{C}$ for $12 \mathrm{~h}$. Supernatants were removed and transferred to fresh tubes. Additional peptides were extracted from the gel by adding 50\% acetonitrile and 1\% TFA and shaking for $10 \mathrm{~min}$. The supernatants were combined and dried. The dried samples were reconstituted in $0.1 \%$ formic acid for mass spectrometry analysis.

\section{Proteomics analysis by nano-LC-MS/MS}

Peptides were analyzed on a Q-Exactive Orbitrap Mass Spectrometer attached to an Easy-nLC (Thermo Fisher Scientific) at $400 \mathrm{nl} / \mathrm{min}$. Peptides were eluted with a $25 \mathrm{~min}$ gradient of 5 to $32 \% \mathrm{ACN}$ followed by $5 \mathrm{~min} 90 \% \mathrm{ACN}$ and $0.1 \%$ formic acid. The data-dependent acquisition mode with a dynamic exclusion of $45 \mathrm{~s}$ was used for analysis. A full MS scan range of 350 to $1200 \mathrm{~m} / \mathrm{z}$ was collected, with a resolution of $70 \mathrm{~K}$, maximum injection time of $50 \mathrm{~ms}$, and automatic gain control (AGC) of $1 \times 10^{6}$. A series of MS2 scans were then acquired for the top 12 most abundant ions of the MS1 scans. Ions were filtered with charge 2 to 4 . An isolation window of $2 \mathrm{~m} / \mathrm{z}$ was used with the quadruple isolation mode. Ions were fragmented using higher-energy collisional dissociation (HCD), with a collision energy of $27 \%$. Orbitrap detection was used with a scan range of 140 to $2000 \mathrm{~m} / \mathrm{z}$, resolution of $30 \mathrm{~K}$, maximum injection time of $54 \mathrm{~ms}$, and AGC of 50,000. All the samples were analyzed using a multiplexed parallel reaction monitoring (PRM) method based on a scheduled inclusion list containing the target precursor ions. Full MS scans were 


\section{Novel mammalian cell expression and purification method}

acquired on the Orbitrap from 350 to $1200 \mathrm{~m} / \mathrm{z}$ at a resolution of 60,000, using an AGC of 50,000. The minimum threshold was set to 100,000 ion counts. Precursor ions were fragmented with the quadrupole using an isolation width of $2 \mathrm{~m} / \mathrm{z}$ units, a maximum injection time of $50 \mathrm{~ms}$, and AGC of 10,000.

\section{Proteomics MS data analysis including peptide identification and quantification}

The mass spectrometry raw spectra were processed using Proteome Discoverer version 2.3 (Thermo Fisher Scientific). The spectra were searched against a database of target protein sequences using default setting: precursor mass tolerance of $10 \mathrm{ppm}$, fragment mass tolerance of $0.02 \mathrm{Da}$, enzymes-specific cleavage sites, and up to two missed cleavages. Cysteine carbamidomethylation was set as a fixed modification, while methionine oxidation and N-terminal or lysine acetylation were set as variable modifications. The search results were filtered using the target-decoy approach with the false discovery rate (FRD) cutoff of $<1 \%$ at the peptide and protein levels. To identify PTMs, the raw data were reprocessed with the program MetaMorepheus version 0.0.316 optimized for PTM analysis (91). The calibrate, G-PTM-D, and search tasks of the program were used with default parameters, including default fixed and variable modifications, precursor mass tolerance of $5 \mathrm{ppm}$, enzyme specific cleavage, up to two missed cleavages, and an FRD cutoff of $<1 \%$ at the peptide and protein levels.

\section{Cosedimentation assays}

Actin filaments at a fixed concentration of $7 \mu \mathrm{M}$ were incubated for $30 \mathrm{~min}$ at $25^{\circ} \mathrm{C}$ with increasing Tpm concentrations in cosedimentation buffer $(20 \mathrm{mM}$ HEPES pH 7.5, $200 \mathrm{mM} \mathrm{NaCl}, 5 \mathrm{mM} \mathrm{MgCl}_{2}$ ) and a total volume of $100 \mu \mathrm{l}$. The reactions were centrifuged for $30 \mathrm{~min}$ at $80,000 \mathrm{rpm}(280,000$ rcf) to pellet F-actin and any bound Tpm (Fig. $3 A$ and Fig. S4). For each Tpm protein, the cosedimentation experiments were repeated three or four times (Fig. S3). After centrifugation, $100 \mu \mathrm{l}$ of the supernatants were mixed with $25 \mu \mathrm{l}$ of $4 \times$ Laemmli SDS-PAGE loading buffer (S fraction). Pellets were gently washed with $100 \mu \mathrm{l}$ of cosedimentation buffer, to remove any remaining soluble protein, and resuspended in $100 \mu \mathrm{l}$ of cosedimentation buffer and mixed with $25 \mu \mathrm{l}$ of $4 \times$ Laemmli SDS-PAGE loading buffer ( $P$ fraction). Identical volumes $(7 \mu \mathrm{l})$ of the $\mathrm{P}$ and $\mathrm{S}$ fractions were analyzed on SDSPAGE gels, Coomassie-stained, and the bands were densitometrically quantified with the program Image Lab (BIO-RAD). For experiments using purified rabbit skeletal muscle Tpm, the intensities of the two bands (isoforms Tpm1.1 and Tpm2.2) were summed. In the absence of actin filaments, all the Tpm isoforms were found in the $\mathrm{S}$ fraction, whereas most of the actin was found in the $\mathrm{P}$ fraction with or without Tpm (Fig. $3 \mathrm{~A}$ and Fig. S4). In the presence of actin filaments, Tpm cosedimented with actin to different degrees. The free Tpm concentration (plotted on the $\mathrm{x}$-axis) was determined by dividing the intensity of the Tpm band in the $\mathrm{S}$ fraction by the sum of the intensities of the Tpm bands in the $\mathrm{S}$ and $\mathrm{P}$ fractions and multiplied by the total Tpm concentration. The fraction of
Tpm bound in the pellet was determined by dividing the intensities of the Tpm and actin bands in the pellet fraction. According to a commonly used convention $(92,93)$, we plotted on the $\mathrm{y}$-axis the fraction of maximal Tpm binding to F-actin, determined by normalizing the ratio of the intensities of the Tpm and actin bands in the pellet fraction to the plateau value for each Tpm sample (i.e., the maximum fraction of Tpm/actin in the pellet fraction). This allows to compare side by side Tpm isoforms with different F-actin-binding affinities by plotting the fraction of maximal binding versus the free Tpm concentration and fitting the data to a Hill equation for cooperative binding. We used the program Prism 9.0.0 (GraphPad) for fitting with a "specific binding with Hill slope" model, which yielded the apparent dissociation constant $\left(K_{\text {app }}\right)$ and Hill coefficient for each interaction (Fig. 3F).

\section{Detection of nonmuscle Tpm heterodimers}

Expi293F cell cultures $(25 \mathrm{ml})$ expressing pairs of Tpm isoforms using the IRES-containing vector pJC8 (see above) were resuspended in FLAG lysis buffer (phosphate buffered saline, $1 \mathrm{mM}$ EDTA, $1 \mathrm{mM}$ PMFS, and 0.5\% Triton X-100) and lysed by three cycles of freeze-thaw in liquid nitrogen. Lysates were clarified at $4{ }^{\circ} \mathrm{C}$ by centrifugation at $48,000 \mathrm{~g}$ for $20 \mathrm{~min}$ and incubated with $100 \mu \mathrm{l}$ of anti-DYKDDDDK (FLAG) resin (Thermo Fisher Scientific) for $2 \mathrm{~h}$ at $4{ }^{\circ} \mathrm{C}$. The resin was washed five times with $200 \mu \mathrm{l}$ lysis buffer. FLAG-tagged proteins were released from the resin by incubation with buffer supplemented with $50 \mathrm{mM}$ FLAG peptide (Genscript) for $30 \mathrm{~min}$.

For boiling and refolding experiments, the proteins purified from the FLAG resin were heated for $10 \mathrm{~min}$ at $90^{\circ} \mathrm{C}$ and then allowed to cool to $25{ }^{\circ} \mathrm{C}$ over $30 \mathrm{~min}$. The proteins were incubated for $2 \mathrm{~h}$ at $4{ }^{\circ} \mathrm{C}$ with $100 \mu \mathrm{l}$ of V5 resin, washed five times with $200 \mu \mathrm{l}$ lysis buffer, and directly used for analysis. Equal volumes $(100 \mu \mathrm{l})$ of lysate, flowthrough, first wash, last wash, FLAG elution, and V5 resin-bound proteins were mixed with $25 \mu \mathrm{l}$ of $4 \times$ Laemmli SDS-PAGE loading buffer and analyzed by Western blotting using primary FLAG (Santa Cruz Biotechnology) and V5 antibodies (Thermo Fisher Scientific). Secondary ECL-linked anti-mouse and anti-rabbit antibodies (Cytiva Life Sciences) were applied and the blots were imaged using a Pierce ECL Plus Western Blotting Substrate (Thermo Fisher Scientific) on a G:BOX Chemi XR5 imager (Syngene).

\section{Data availability}

The two vectors developed here were deposited to the Addgene plasmid repository (www.addgene.org) with plasmid ID numbers 170756 and 170757. Raw proteomics data files were deposited to the MassIVE data repository with the accession number MSV000087517 (https://doi.org/10.25345/ C5K528). All remaining data are contained within the article.

Supporting information-This article contains supporting information.

Acknowledgments-We thank Pekka Lappalainen for the gift of vectors for the bacterial expression of Tpm isoforms. We thank the 
Quantitative Proteomics Resource Core at the Perelman School of Medicine (University of Pennsylvania), Hyoungjoo Lee, Joseph Cesare, and Michael Gilbert for help with the proteomics data acquisition and analysis. We thank Michael Shortreed for help with the use of the program MetaMorpheus.

Author contributions-P. J. C., K. R. B., and R. D. conceptualization; P. J. C. and K. R. B. data curation; P. J. C. and K. R. B. formal analysis; P. J. C. and R. D. funding acquisition; P. J. C., K. R. B., and R. D. investigation; P. J. C. and K. R. B. methodology; P. J. C., K. R. B., and R. D. project administration; P. J. C., K. R. B., and R. D. resources; P. J. C. and K. R. B. software; P. J. C. and R. D. supervision; P. J. C. and K. R. B. validation; P. J. C., K. R. B., and R. D. visualization; P. J. C., K. R. B., and R. D. writing-original draft; P. J. C., K. R. B., and R. D. writing-review and editing.

Funding and additional information-This work was supported by National Institutes of Health Grants R01 GM073791 and R01 MH087950 to R. D. and T32 AR053461 and F31 HL156431 to P. J. C. The content is solely the responsibility of the authors and does not necessarily represent the official views of the National Institutes of Health.

Conflict of interest-The authors declare that they have no conflicts of interest with the contents of this article.

Abbreviations-The abbreviations used are: CBD, chitin-binding domain; CMV, cytomegalovirus promoter; FDR, false discovery rate; HCD, higher-energy collisional dissociation; HEK, human embryonic kidney; PTM, posttranslational modification; Tpm, tropomyosin; WPRE, woodchuck hepatitis virus posttranscriptional regulatory element.

\section{References}

1. Waugh, D. S. (2011) An overview of enzymatic reagents for the removal of affinity tags. Protein Expr. Purif. 80, 283-293

2. Khoury, G. A., Baliban, R. C., and Floudas, C. A. (2011) Proteome-wide post-translational modification statistics: Frequency analysis and curation of the swiss-prot database. Sci. Rep. 1, 90

3. Bah, A., and Forman-Kay, J. D. (2016) Modulation of intrinsically disordered protein function by post-translational modifications. J. Biol. Chem. $291,6696-6705$

4. Hunter, T. (2009) Tyrosine phosphorylation: Thirty years and counting. Curr. Opin. Cell Biol. 21, 140-146

5. Johnson, L. N., and Lewis, R. J. (2001) Structural basis for control by phosphorylation. Chem. Rev. 101, 2209-2242

6. Walsh, C. T., Garneau-Tsodikova, S., and Gatto, G. J., Jr. (2005) Protein posttranslational modifications: The chemistry of proteome diversifications. Angew. Chem. Int. Ed. Engl. 44, 7342-7372

7. Amann, T., Schmieder, V., Faustrup Kildegaard, H., Borth, N., and Andersen, M. R. (2019) Genetic engineering approaches to improve posttranslational modification of biopharmaceuticals in different production platforms. Biotechnol. Bioeng. 116, 27782796

8. Brown, C. W., Sridhara, V., Boutz, D. R., Person, M. D., Marcotte, E. M., Barrick, J. E., and Wilke, C. O. (2017) Large-scale analysis of posttranslational modifications in E. coli under glucose-limiting conditions. BMC Genomics 18, 301

9. Higel, F., Seidl, A., Sorgel, F., and Friess, W. (2016) N-glycosylation heterogeneity and the influence on structure, function and pharmacokinetics of monoclonal antibodies and Fc fusion proteins. Eur. J. Pharm. Biopharm. 100, 94-100

10. McKenzie, E. A., and Abbott, W. M. (2018) Expression of recombinant proteins in insect and mammalian cells. Methods 147, 40-49
11. Tang, H., Wang, S., Wang, J., Song, M., Xu, M., Zhang, M., Shen, Y., Hou, J., and Bao, X. (2016) N-hypermannose glycosylation disruption enhances recombinant protein production by regulating secretory pathway and cell wall integrity in Saccharomyces cerevisiae. Sci. Rep. 6, 25654

12. Weis, B. L., Guth, N., Fischer, S., Wissing, S., Fradin, S., Holzmann, K. H., Handrick, R., and Otte, K. (2018) Stable miRNA overexpression in human CAP cells: Engineering alternative production systems for advanced manufacturing of biologics using miR-136 and miR-3074. Biotechnol. Bioeng. 115, 2027-2038

13. Dunn, A. Y., Melville, M. W., and Frydman, J. (2001) Review: Cellular substrates of the eukaryotic chaperonin TRiC/CCT. J. Struct. Biol. 135, $176-184$

14. Liu, Z., Tyo, K. E., Martinez, J. L., Petranovic, D., and Nielsen, J. (2012) Different expression systems for production of recombinant proteins in Saccharomyces cerevisiae. Biotechnol. Bioeng. 109, 1259-1268

15. Resnicow, D. I., Deacon, J. C., Warrick, H. M., Spudich, J. A., and Leinwand, L. A. (2010) Functional diversity among a family of human skeletal muscle myosin motors. Proc. Natl. Acad. Sci. U. S. A. 107, 1053-1058

16. Thomas, J. A., and Tate, C. G. (2014) Quality control in eukaryotic membrane protein overproduction. J. Mol. Biol. 426, 4139-4154.

17. Brault, V., Reedy, M. C., Sauder, U., Kammerer, R. A., Aebi, U., and Schoenenberger, C. (1999) Substitution of flight muscle-specific actin by human (beta)-cytoplasmic actin in the indirect flight muscle of Drosophila. J. Cell Sci. 112, 3627-3639

18. Sliwinska, M., Skorzewski, R., and Moraczewska, J. (2008) Role of actin Cterminus in regulation of striated muscle thin filament. Biophys. J. 94, $1341-1347$

19. Chant, A., Kraemer-Pecore, C. M., Watkin, R., and Kneale, G. G. (2005) Attachment of a histidine tag to the minimal zinc finger protein of the Aspergillus nidulans gene regulatory protein AreA causes a conformational change at the DNA-binding site. Protein Expr. Purif. 39, 152-159

20. Suh-Lailam, B. B., and Hevel, J. M. (2009) Efficient cleavage of problematic tobacco etch virus (TEV)-protein arginine methyltransferase constructs. Anal. Biochem. 387, 130-132

21. Wu, J., and Filutowicz, M. (1999) Hexahistidine (His6)-tag dependent protein dimerization: A cautionary tale. Acta Biochim. Pol. 46, 591-599

22. Gunning, P. W., Hardeman, E. C., Lappalainen, P., and Mulvihill, D. P. (2015) Tropomyosin - master regulator of actin filament function in the cytoskeleton. J. Cell Sci. 128, 2965-2974.

23. Geeves, M. A., Hitchcock-DeGregori, S. E., and Gunning, P. W. (2015) A systematic nomenclature for mammalian tropomyosin isoforms. J. Muscle Res. Cell Motil. 36, 147-153

24. Meiring, J. C. M., Bryce, N. S., Wang, Y., Taft, M. H., Manstein, D. J., Liu Lau, S., Stear, J., Hardeman, E. C., and Gunning, P. W. (2018) Co-polymers of actin and tropomyosin account for a major fraction of the human actin cytoskeleton. Curr. Biol. 28, 2331-2337.e2335

25. Gunning, P. W., Ghoshdastider, U., Whitaker, S., Popp, D., and Robinson, R. C. (2015) The evolution of compositionally and functionally distinct actin filaments. J. Cell Sci. 128, 2009-2019

26. Lehman, W., Hatch, V., Korman, V., Rosol, M., Thomas, L., Maytum, R., Geeves, M. A., Van Eyk, J. E., Tobacman, L. S., and Craig, R. (2000) Tropomyosin and actin isoforms modulate the localization of tropomyosin strands on actin filaments. J. Mol. Biol. 302, 593-606

27. McKillop, D. F., and Geeves, M. A. (1993) Regulation of the interaction between actin and myosin subfragment 1: Evidence for three states of the thin filament. Biophys. J. 65, 693-701

28. Cheng, C., Nowak, R. B., Amadeo, M. B., Biswas, S. K., Lo, W.-K., and Fowler, V. M. (2018) Tropomyosin 3.5 protects the F-actin networks required for tissue biomechanical properties. J. Cell Sci. 131, jcs222042

29. Pleines, I., Woods, J., Chappaz, S., Kew, V., Foad, N., Ballester-Beltrán, J., Aurbach, K., Lincetto, C., Lane, R. M., Schevzov, G., Alexander, W. S., Hilton, D. J., Astle, W. J., Downes, K., Nurden, P., et al. (2017) Mutations in tropomyosin 4 underlie a rare form of human macrothrombocytopenia. J. Clin. Invest. 127, 814-829

30. Kee, A. J., Gunning, P. W., and Hardeman, E. C. (2009) A cytoskeletal tropomyosin can compromise the structural integrity of skeletal muscle. Cell Motil. Cytoskeleton 66, 710-720 
31. Vlahovich, N., Kee, A. J., Van der Poel, C., Kettle, E., Hernandez-Deviez, D., Lucas, C., Lynch, G. S., Parton, R. G., Gunning, P. W., and Hardeman, E. C. (2009) Cytoskeletal tropomyosin Tm5NM1 is required for normal excitation-contraction coupling in skeletal muscle. Mol. Biol. Cell 20, 4.00-409

32. Eppinga, R. D., Li, Y., Lin, J. L., and Lin, J. J. (2006) Tropomyosin and caldesmon regulate cytokinesis speed and membrane stability during cell division. Arch. Biochem. Biophys. 456, 161-174

33. Thoms, J. A., Loch, H. M., Bamburg, J. R., Gunning, P. W., and Weinberger, R. P. (2008) A tropomyosin 1 induced defect in cytokinesis can be rescued by elevated expression of cofilin. Cell Motil. Cytoskeleton 65, 979-990

34. Hook, J., Lemckert, F., Qin, H., Schevzov, G., and Gunning, P. (2004) Gamma tropomyosin gene products are required for embryonic development. Mol. Cell. Biol. 24, 2318-2323

35. Hook, J., Lemckert, F., Schevzov, G., Fath, T., and Gunning, P. (2011) Functional identity of the gamma tropomyosin gene: Implications for embryonic development, reproduction and cell viability. Bioarchitecture 1, 49-59

36. Dalby-Payne, J. R., O'Loughlin, E. V., and Gunning, P. (2003) Polarization of specific tropomyosin isoforms in gastrointestinal epithelial cells and their impact on CFTR at the apical surface. Mol. Biol. Cell 14, 4365-4375

37. Dufour, C., Weinberger, R. P., and Gunning, P. (1998) Tropomyosin isoform diversity and neuronal morphogenesis. Immunol. Cell Biol. 76, 4.24-429

38. Had, L., Faivre-Sarrailh, C., Legrand, C., and Rabie, A. (1993) The expression of tropomyosin genes in pure cultures of rat neurons, astrocytes and oligodendrocytes is highly cell-type specific and strongly regulated during development. Brain Res. Mol. Brain Res. 18, 77-86

39. Hannan, A. J., Gunning, P., Jeffrey, P. L., and Weinberger, R. P. (1998) Structural compartments within neurons: Developmentally regulated organization of microfilament isoform mRNA and protein. Mol. Cell. Neurosci. 11, 289-304.

40. Heald, R. W., and Hitchcock-DeGregori, S. E. (1988) The structure of the amino terminus of tropomyosin is critical for binding to actin in the absence and presence of troponin. J. Biol. Chem. 263, 5254-5259

41. Palm, T., Greenfield, N. J., and Hitchcock-DeGregori, S. E. (2003) Tropomyosin ends determine the stability and functionality of overlap and troponin T complexes. Biophys. J. 84, 3181-3189

42. Maytum, R., Geeves, M. A., and Konrad, M. (2000) Actomyosin regulatory properties of yeast tropomyosin are dependent upon $\mathrm{N}$-terminal modification. Biochemistry 39, 11913-11920

43. Lehman, W., Medlock, G., Li, X. E., Suphamungmee, W., Tu, A. Y., Schmidtmann, A., Ujfalusi, Z., Fischer, S., Moore, J. R., Geeves, M. A., and Regnier, M. (2015) Phosphorylation of Ser283 enhances the stiffness of the tropomyosin head-to-tail overlap domain. Arch. Biochem. Biophys. $571,10-15$

44. Palani, S., Köster, D. V., Hatano, T., Kamnev, A., Kanamaru, T., Brooker, H. R., Hernandez-Fernaud, J. R., Jones, A. M. E., Millar, J. B. A., Mulvihill, D. P., and Balasubramanian, M. K. (2019) Phosphoregulation of tropomyosin is crucial for actin cable turnover and division site placement. J. Cell Biol. 218, 3548-3559

45. Rajan, S., Jagatheesan, G., Petrashevskaya, N., Biesiadecki, B. J., Warren, C. M., Riddle, T., Liggett, S., Wolska, B. M., Solaro, R. J., and Wieczorek, D. F. (2019) Tropomyosin pseudo-phosphorylation results in dilated cardiomyopathy. J. Biol. Chem. 294, 2913-2923

46. Rao, V. S., Marongelli, E. N., and Guilford, W. H. (2009) Phosphorylation of tropomyosin extends cooperative binding of myosin beyond a single regulatory unit. Cell Motil. Cytoskeleton 66, 10-23

47. Brooker, H. R., Geeves, M. A., and Mulvihill, D. P. (2016) Analysis of biophysical and functional consequences of tropomyosin-fluorescent protein fusions. FEBS Lett. 590, 3111-3121

48. Frye, J., Klenchin, V. A., and Rayment, I. (2010) Structure of the tropomyosin overlap complex from chicken smooth muscle: Insight into the diversity of N-terminal recognition. Biochemistry 49, 4908-4920

49. Monteiro, P. B., Lataro, R. C., Ferro, J. A., and Reinach Fde, C. (1994) Functional alpha-tropomyosin produced in Escherichia coli. A dipeptide extension can substitute the amino-terminal acetyl group. J. Biol. Chem. 269, 10461-10466
50. Johnson, M., Coulton, A. T., Geeves, M. A., and Mulvihill, D. P. (2010) Targeted amino-terminal acetylation of recombinant proteins in E. coli. PLoS One 5, e15801

51. Wingfield, P. T. (2017) N-terminal methionine processing. Curr. Protoc. Protein Sci. 88, 6.14.1-6.14.3

52. Schmid, A. W., Fauvet, B., Moniatte, M., and Lashuel, H. A. (2013) Alphasynuclein post-translational modifications as potential biomarkers for Parkinson disease and other synucleinopathies. Mol. Cell. Proteomics 12, 3543-3558

53. Zhang, J., Li, X., and Li, J. D. (2019) The roles of post-translational modifications on alpha-synuclein in the pathogenesis of Parkinson's diseases. Front. Neurosci. 13, 381

54. Backliwal, G., Hildinger, M., Hasija, V., and Wurm, F. M. (2008) Highdensity transfection with HEK-293 cells allows doubling of transient titers and removes need for a priori DNA complex formation with PEI. Biotechnol. Bioeng. 99, 721-727

55. Liu, C., Dalby, B., Chen, W., Kilzer, J. M., and Chiou, H. C. (2008) Transient transfection factors for high-level recombinant protein production in suspension cultured mammalian cells. Mol. Biotechnol. 39, $141-153$

56. Shah, N. H., and Muir, T. W. (2014) Inteins: Nature's gift to protein chemists. Chem. Sci. 5, 44.6-4.61

57. Southworth, M. W., Amaya, K., Evans, T. C., Xu, M. Q., and Perler, F. B. (1999) Purification of proteins fused to either the amino or carboxy terminus of the Mycobacterium xenopi gyrase A intein. Biotechniques 27, $110-114,116,118-120$

58. Aksnes, H., Drazic, A., Marie, M., and Arnesen, T. (2016) First things first: Vital protein marks by $\mathrm{N}$-terminal acetyltransferases. Trends Biochem. Sci. 41, 746-760

59. Gray, D. (2001) Overview of protein expression by mammalian cells. Curr. Protoc. Protein Sci. Chapter 5(1):Unit5.9

60. Khan, K. H. (2013) Gene expression in mammalian cells and its applications. Adv. Pharm. Bull. 3, 257-263

61. Tokmakov, A. A., Kurotani, A., Takagi, T., Toyama, M., Shirouzu, M., Fukami, Y., and Yokoyama, S. (2012) Multiple post-translational modifications affect heterologous protein synthesis. J. Biol. Chem. 287, 2710627116

62. Wood, D. W., and Camarero, J. A. (2014) Intein applications: From protein purification and labeling to metabolic control methods. J. Biol. Chem. 289, 14512-14519

63. Telenti, A., Southworth, M., Alcaide, F., Daugelat, S., Jacobs, W. R., Jr., and Perler, F. B. (1997) The Mycobacterium xenopi GyrA protein splicing element: Characterization of a minimal intein. J. Bacteriol. 179, 6378-6382

64. Hirata, R., Ohsumk, Y., Nakano, A., Kawasaki, H., Suzuki, K., and Anraku, Y. (1990) Molecular structure of a gene, VMA1, encoding the catalytic subunit of $\mathrm{H}(+)$-translocating adenosine triphosphatase from vacuolar membranes of Saccharomyces cerevisiae. J. Biol. Chem. 265, $6726-6733$

65. Kane, P. M., Yamashiro, C. T., Wolczyk, D. F., Neff, N., Goebl, M., and Stevens, T. H. (1990) Protein splicing converts the yeast TFP1 gene product to the $69-\mathrm{kD}$ subunit of the vacuolar $\mathrm{H}(+)$-adenosine triphosphatase. Science 250, 651-657

66. Chong, S., Shao, Y., Paulus, H., Benner, J., Perler, F. B., and Xu, M. Q. (1996) Protein splicing involving the Saccharomyces cerevisiae VMA intein. The steps in the splicing pathway, side reactions leading to protein cleavage, and establishment of an in vitro splicing system. J. Biol. Chem. 271, 22159-22168

67. Van Hoof, C., and Goris, J. (2003) Phosphatases in apoptosis: To be or not to be, PP2A is in the heart of the question. Biochim. Biophys. Acta 1640, 97-104

68. Drazic, A., Myklebust, L. M., Ree, R., and Arnesen, T. (2016) The world of protein acetylation. Biochim. Biophys. Acta 1864, 1372-14.01

69. Anderson, J. P., Walker, D. E., Goldstein, J. M., de Laat, R., Banducci, K., Caccavello, R. J., Barbour, R., Huang, J., Kling, K., Lee, M., Diep, L., Keim, P. S., Shen, X., Chataway, T., Schlossmacher, M. G., et al. (2006) Phosphorylation of Ser-129 is the dominant pathological modification of alpha-synuclein in familial and sporadic Lewy body disease. J. Biol. Chem. 281, 29739-29752 
70. Schey, K. L., and Finley, E. L. (2000) Identification of peptide oxidation by tandem mass spectrometry. Acc. Chem. Res. 33, 299-306

71. Hornbeck, P. V., Zhang, B., Murray, B., Kornhauser, J. M., Latham, V., and Skrzypek, E. (2015) PhosphoSitePlus, 2014: Mutations, PTMs and recalibrations. Nucleic Acids Res. 43, D512-520

72. Gateva, G., Kremneva, E., Reindl, T., Kotila, T., Kogan, K., Gressin, L., Gunning, P. W., Manstein, D. J., Michelot, A., and Lappalainen, P. (2017) Tropomyosin isoforms specify functionally distinct actin filament populations in vitro. Curr. Biol. 27, 705-713

73. Bronson, D. D., and Schachat, F. H. (1982) Heterogeneity of contractile proteins. Differences in tropomyosin in fast, mixed, and slow skeletal muscles of the rabbit. J. Biol. Chem. 257, 3937-3944

74. Tobacman, L. S. (2008) Cooperative binding of tropomyosin to actin. Adv. Exp. Med. Biol. 644, 85-94.

75. Wegner, A. (1979) Equilibrium of the actin-tropomyosin interaction. J. Mol. Biol. 131, 839-853

76. Yang, Y. Z., Korn, E. D., and Eisenberg, E. (1979) Cooperative binding of tropomyosin to muscle and Acanthamoeba actin. J. Biol. Chem. 254, $7137-7140$

77. Bicer, S., and Reiser, P. J. (2013) Complex tropomyosin and troponin T isoform expression patterns in orbital and global fibers of adult dog and rat extraocular muscles. J. Muscle Res. Cell Motil. 34, 211-231

78. Sanders, C., Burtnick, L. D., and Smillie, L. B. (1986) Native chicken gizzard tropomyosin is predominantly a beta gamma-heterodimer. J. Biol. Chem. 261, 12774-12778

79. Muthuchamy, M., Grupp, I. L., Grupp, G., O’Toole, B. A., Kier, A. B., Boivin, G. P., Neumann, J., and Wieczorek, D. F. (1995) Molecular and physiological effects of overexpressing striated muscle beta-tropomyosin in the adult murine heart. J. Biol. Chem. 270, 30593-30603

80. Kast, D. J., and Dominguez, R. (2019) Mechanism of IRSp53 inhibition by 14-3-3. Nat. Commun. 10, 483

81. Coulton, A., Lehrer, S. S., and Geeves, M. A. (2006) Functional homodimers and heterodimers of recombinant smooth muscle tropomyosin. Biochemistry 45, 12853-12858
82. Jancso, A., and Graceffa, P. (1991) Smooth muscle tropomyosin coiledcoil dimers. Subunit composition, assembly, and end-to-end interaction. J. Biol. Chem. 266, 5891-5897

83. Graceffa, P. (1992) Heat-treated smooth muscle tropomyosin. Biochim. Biophys. Acta 1120, 205-207

84. Lehrer, S. S., and Qian, Y. (1990) Unfolding/refolding studies of smooth muscle tropomyosin. Evidence for a chain exchange mechanism in the preferential assembly of the native heterodimer. J. Biol. Chem. 265, 11341138

85. Heeley, D. H. (2013) Phosphorylation of tropomyosin in striated muscle. J. Muscle Res. Cell Motil. 34, 233-237

86. Ribolow, H., and Barany, M. (1977) Phosphorylation of tropomyosin in live frog muscle. Arch. Biochem. Biophys. 179, 718-720

87. Johnson, P., and Smillie, L. B. (1977) Polymerizability of rabbit skeletal tropomyosin: Effects of enzymic and chemical modifications. Biochemistry 16, 2264-2269

88. Schmidt, W., Madan, A., Foster, D. B., and Cammarato, A. (2020) Lysine acetylation of $\mathrm{F}$-actin decreases tropomyosin-based inhibition of actomyosin activity. J. Biol. Chem. 295, 15527-15539

89. Jansen, S., and Goode, B. L. (2019) Tropomyosin isoforms differentially tune actin filament length and disassembly. Mol. Biol. Cell 30, 671-679

90. Gormal, R., Valmas, N., Fath, T., and Meunier, F. (2017) A role for tropomyosins in activity-dependent bulk endocytosis? Mol. Cell. Neurosci. 84, $112-118$

91. Solntsev, S. K., Shortreed, M. R., Frey, B. L., and Smith, L. M. (2018) Enhanced global post-translational modification discovery with MetaMorpheus. J. Proteome Res. 17, 1844-1851

92. Moraczewska, J., Greenfield, N. J., Liu, Y., and Hitchcock-DeGregori, S. E. (2000) Alteration of tropomyosin function and folding by a nemaline myopathy-causing mutation. Biophys. J. 79, 3217-3225

93. Rynkiewicz, M. J., Prum, T., Hollenberg, S., Kiani, F. A., Fagnant, P. M., Marston, S. B., Trybus, K. M., Fischer, S., Moore, J. R., and Lehman, W. (2017) Tropomyosin must interact weakly with actin to effectively regulate thin filament function. Biophys. J. 113, 2444-2451 